\title{
A note on compactness-type properties with respect to Lorentz norms of bounded subsets of a Sobolev Space
}

by

\author{
Sergio SOLIMINI \\ Dipartimento di Matematica, \\ Universitá di Lecce.
}

\begin{abstract}
Some properties of "concentration-compactness type" are proved to the aim of characterizing the behaviour of bounded sequences of functions in a Sobolev Space with respect to Lorentz norms. Such properties are shown to exist as far as the embedding is not optimal with respect to the secundary index.
\end{abstract}

RÉSUMÉ. - Des propriétés "du type concentration-compacité " sont démontrées dans le but de caractériser le comportement de suites bornées de fonctions dans un Espace de Sobolev relativement aux normes de Lorentz. Ces propriétés sont prouvées tant que le plongement n'est pas optimal par rapport à l'exposant secondaire.

\section{INTRODUCTION}

In [2] some partial differential equations are studied which involve singular coefficients. A variational approach to such problems requires the use of the Sobolev's Embedding in Lorentz Spaces and some compactness properties like those introduced by P. L. Lions in [4]-[7] or those stated

Strada provinciale Lecce-Arnesano, 73100 Lecce, Italy.

Annales de l'Institut Henri Poincaré - Analyse non linéaire - 0294-1449 Vol. $12 / 95 / 03 / \$ 4.00 /$ 
in [11]. The methods in [4]-[7] cover this case, which is in fact treated in [7, II4], however the analysis of the compactness properties of Sobolev's Embedding in the context of Lorentz Spaces presents some interesting features which we want to point out in this note. In fact we shall show the two following facts:

a) some compactness properties are an easy consequence of Sobolev's Embedding in Lorentz Spaces, provided the embedding is not the optimal one. In fact, a straightforward interpolation argument reduces the proof to the case of weak $L^{p}$ spaces where a simple device can be used;

b) the same properties do not hold for the case of the optimal embedding, as one can see from an easy counterexample and from the analysis of some concrete problems in which such embedding is involved and which have no solution.

So we are in an analogous situation to that of the classical Rellich Theorem: compactness results are available as far as the embedding is not optimal. The analogy cannot be appreciated if one only looks to the usual $L^{p}$ structure.

Any further comment will be postponed after a preliminary explanation of the notions about Lorentz Spaces and of the notation that the reader will be supposed to know. This will be done in the first section in which the above mentioned compactness properties will also be specified. We shall give them in three equivalent statements: an alternative theorem (Theorem 1) which states that a bounded sequence in the Sobolev Space must be infinitesimal in the Lorentz Space or it can reach a nonzero weak limit modulo a suitable change of scale of every term, a structure theorem (Theorem 2) which shows how such a sequence can be approximated and a compact embedding theorem (Theorem 3) which characterizes with properties related to the changes of scale the bounded subsets of the Sobolev Space which are embedded as precompact subsets in the Lorentz Space.

The second section will be devoted to the proof of the theorems.

In the third section we shall mention the particular cases in which the conclusions can be furtherly specified and we shall show the counterexample for the optimal case.

For new applications we refer the reader to [2], however we point out once more that they could already be treated with the methods in [7]. The aim of this note only consists in suggesting a different point of view and in observing how the strict cases of the concentration-compactness inequalities correspond in some applications to the fact that nonoptimal embeddings are involved. 


\section{NOTATION AND STATEMENT OF THE RESULTS}

We shall only recall what the reader has to know about Lorentz Spaces in order to follow this note. We shall even omit to give the classical definition, which requires some discussion and which can be found, for istance, in [9], while we shall list in a purely operative way the essential properties of those spaces involved with the refinement of Sobolev's Embedding.

A Lorentz Space $L(p, q)\left(\mathbb{R}^{N}\right)$ is a space of measurable functions affected by two indexes $p$ and $q$ which are two positive real numbers, $1 \leq p, q \leq \infty$, like the indexes which determine the usual $L^{p}$ spaces. The index $p$ is called principal index and the index $q$ is called secundary index.

A monotonicity property holds with respect to the secundary index, in fact, if $q_{1}<q_{2}$, then we have $L\left(p, q_{1}\right) \subset L\left(p, q_{2}\right)$. So the strongest case of a Lorentz Space with principal index $p$ is the space $L(p, 1)$, while the weakest one is $L(p, \infty)$. The most familiar one is the intermediate case given by $q=p$, in fact the space $L(p, p)$ is equivalent to the classical $L^{p}$ space. The already mentioned weakest space $L(p, \infty)$ is on the other hand equivalent to the weak $L^{p}$ space, or Marcinkiewicz Space, namely the space $M^{p}$ consisting of the measurable functions $u$ such that one can find a constant $c$ in such a way that

$$
\forall X \subset \mathbb{R}^{N} \quad \int_{X}|u| \leq c|X|^{1-\frac{1}{p}},
$$

where $|X|$ denotes the Lebesgue measure of the set $X$. The least value of the constant $c$ such that (1) holds will be called Marcinkiewicz norm of $u$.

The knowledge of these two particular cases allows an alternative definition of the $L(p, q)$ spaces by interpolation. If $p \leq q$, we shall denote by $L(p, q)$ the space of the functions $u$ such that $u=u_{1}^{\frac{p}{q}} u_{2}^{1-\frac{p}{q}}$, with $u_{1} \in L^{p}$ and $u_{2} \in M^{p}$. If $q \leq p$, we shall denote by $L(p, q)$ the space of the functions $u$ such that for every $v \in M^{r}$, with $r$ such that $\frac{1}{q}=\frac{1}{p}+\frac{1}{r}, u v \in L^{q}$. The spaces will be equipped with the corresponding interpolation norms.

No monotonicity properties can of course hold with respect to the principal index, as one sees from the particular case of the $L^{p}$ spaces. On the other hand, the secundary index affects the space only in an "infinitesimal" way with respect to the first one. In fact, if some function belongs to the Marcinkiewicz spaces $M^{p-\varepsilon}$ and $M^{p+\varepsilon}$, for a positive value of $\varepsilon$, then it also belongs to $L(p, 1)$, the strongest Lorentz space with principal index $p$. An interpolation property also holds with respect to the secundary index, if a sequence converges in $L\left(p, q_{1}\right)$ and is bounded in $L\left(p, q_{2}\right)$ then it converges in $L(p, q)$, for every index $q$ included between $q_{1}$ and $q_{2}$.

Vol. $12, n^{\circ} 3-1995$. 
A property which we are going to use is the appropriate case of the Hölder Inequality which states that the duality product of two functions is bounded by a constant times the product of the norms of the two functions in two respective "conjugate" Lorentz Spaces $L\left(p_{1}, q_{1}\right)$ and $L\left(p_{2}, q_{2}\right)$, where "conjugate" means that the two pairs of indexes satisfy the relations $\frac{1}{p_{1}}+\frac{1}{p_{2}}=\frac{1}{q_{1}}+\frac{1}{q_{2}}=1$. All the above properties can be easily deduced from the definition.

From now on we shall assume that a positive real number $p, 1 \leq p$, has been given. We shall consider the Sobolev Space $H^{1, p}\left(\mathbb{R}^{N}\right)$ equipped with the "homogeneous" norm which associates to a function the $L^{p}$-norm of its gradient. So the only $L^{q}$ space in which $H^{1, p}\left(\mathbb{R}^{N}\right)$ is embedded is $L^{p^{*}}\left(\mathbb{R}^{N}\right)$, as one can check by using the invariance of the two norms with respect to the same type of the rescalings defined below.

However the embedding in $L^{p^{*}}$ is not the optimal one, if one looks to the richer structure given by Lorentz Spaces. In fact one can show that $H^{1, p}$ is embedded in the space $L\left(p^{*}, p\right)$ which is stronger than $L\left(p^{*}, p^{*}\right)=L^{p^{*}}$. This follows immediately from the convolution rule in Lorentz Spaces [9] and an elementary estimate like the inequality (4) below. A very short and simple alternative proof of this fact, which starts from the definition of Lorentz Spaces and uses a symmetrization argument, can be found in the first few lines of the appendix of [1]. The optimality of $L\left(p^{*}, p\right)$ is in particular proved by the examples in the last section of this note.

We shall call rescaling (of the variable) of center $x_{0}$ and modulus $\lambda$ the affine function $\rho$ which sends a point $x$ of $\mathbb{R}^{N}$ in the point $x_{0}+\lambda\left(x-x_{0}\right)$. The center $x_{0}$ and the modulus $\lambda$ are of course uniquely determined by the rescaling $\rho$ unless $\lambda=1$, namely $\rho$ is the identity map. In order to unify the terminology and to have a stable class with respect to the function product, we shall include among the rescalings also the translations, which are the product of two rescalings with inverse moduli and different centers. In such a case there is no center, the modulus is of course 1 and the function is determined by the translation vector. This discussion is needed for the following definitions. If $\left(\rho_{n}\right)_{n \in \mathbb{N}}$ is a sequence of rescalings we shall say that it is diverging by concentration if the corresponding sequence of the moduli diverges to $+\infty$. On the contrary, we shall say that it is diverging by vanishing if the corresponding sequence of the moduli converges to zero and that it is diverging by translation if the corresponding sequence of the moduli is bounded and bounded away from zero and the corresponding sequence of the centers or of the translation vectors is diverging. We shall say that the sequence of rescalings is diverging if one of the above three cases is verified. Two sequences of rescalings $\left(\rho_{n}\right)_{n \in \mathbb{N}}$ and $\left(\rho_{n}^{1}\right)_{n \in \mathbb{N}}$ will 
said to be mutually diverging if the sequence $\left(\left(\rho_{n}\right)^{-1} \circ \rho_{n}^{1}\right)_{n \in \mathbb{N}}$ is diverging.

From now on we shall assume that the index $p$ is given. If $\rho$ is a rescaling with modulus $\lambda$ and $u$ is a function defined on $\mathbb{R}^{N}$, we shall indicate the function $\lambda^{\alpha} u \circ \rho$ as the rescaled function $u$ by $\rho$ and we shall denote it by the symbol $\rho(u)$. We shall take $\alpha=\frac{N-p}{p}=\frac{N}{p^{*}}$ in order to keep invariant the $H^{1, p}$-norm and the $L\left(p^{*}, q\right)$-norm, for every choice of $q$. We shall also consider the "dual" rescaling, defined as above but with the choice $\alpha=\frac{N+p^{\prime}}{p^{\prime}}=\frac{N}{\left(p^{*}\right)^{\prime}}$ and we shall denote by $\rho^{\prime}(u)$ the function $u$ rescaled by the dual rescaling $\rho^{\prime}$ induced by a rescaling of the variable $\rho$. We note that the dual rescaling $\rho^{\prime}$ leaves invariant the $H^{-1, p^{\prime}}$-norm and the $L^{p^{* \prime}}$-norm.

We shall transfer to the rescalings of the functions the same terminology which we have introduced for the rescalings of the variable. Note that, if $\rho$ is a rescaling and if $u$ and $v$ are two functions which are respectively in $H^{1, p}$ and in $H^{-1, p^{\prime}}$ or respectively in $L\left(p^{*}, q\right)$ and $L\left(\left(p^{*}\right)^{\prime}, q^{\prime}\right)$, then we have

$$
\int_{R^{N}} \rho^{-1}(u) v=\int_{\mathbf{R}^{N}} u \rho^{\prime}(v)
$$

and that a sequence of rescalings $\left(\rho_{n}\right)_{n \in \mathbb{N}}$ is diverging if and only if, for every $u$ in $H^{1, p}$ or, equivalently, for a fixed $u$ in $H^{1, p} \backslash\{0\}$, the sequence $\left(\rho_{n}(u)\right)_{n \in \mathbb{N}}$ weakly converges to zero in $L^{p^{*}}$. Moreover it is diverging by concentration if such a convergence is strong in $L^{q}$, for every $q<p^{*}$ such that $u \in L^{q}$ and it is diverging by vanishing if this happens for $q>p^{*}$.

The above introduced rescalings give an obvious reason for which, for any value of $q$, the embedding of $H^{1, p}$ in $L\left(p^{*}, q\right)$ cannot be compact. In fact, if $u$ is any given nonzero element in $H^{1, p}$ and if $\left(\rho_{n}\right)_{n \in \mathbb{N}}$ is any diverging sequence of rescalings, then $\left(\rho_{n}(u)\right)_{n \in \mathbb{N}}$ has a constant norm in $H^{1, p}$ and in $L\left(p^{*}, q\right)$ while, on the other hand, it weakly converges to zero.

The following statements prove that the cases of the above situation, combined in all the possible ways, are the only possible obstruction to the compactness in $L\left(p^{*}, q\right)$, for $q>p$, of the bounded subsets of $H^{1, p}$.

THEOREM 1. - Let $\left(u_{n}\right)_{n \in \mathbb{N}}$ be a given bounded sequence of functions in $H^{1, p}\left(\mathbb{R}^{N}\right)$, with the index $p$ strictly smaller than $N$.

Then we are in one of the two following alternatives:

a) $\left(u_{n}\right)_{n \in \mathbb{N}}$ converges to zero in $L\left(p^{*}, q\right)\left(\mathbb{R}^{N}\right)$ for every index $q$ strictly greater than $p$.

b) There exists a sequence $\left(\rho_{n}\right)_{n \in \mathbb{N}}$ of rescalings such that the sequence of rescaled functions $\left(\rho_{n}\left(u_{n}\right)\right)_{n \in \mathbb{N}}$ has a nonzero weak limit point (in $\left.L^{p^{*}}\left(\mathbb{R}^{N}\right)\right)$. 
THEOREM 2. - Let $\left(u_{n}\right)_{n \in \mathbb{N}}$ be a given bounded sequence of functions in $H^{1, p}\left(\mathbb{R}^{N}\right)$, with the index $p$ satisfing $1<p<N$. Then, replacing $\left(u_{n}\right)_{n \in \mathbb{N}}$ with a suitable subsequence, we can find a sequence of functions $\left(\phi_{i}\right)_{i \in \mathbb{N}}$ belonging to $H^{1, p}\left(\mathbb{R}^{N}\right)$ and, in correspondence of any index $n$, we can find a sequence of rescalings $\left(\rho_{n}^{i}\right)_{i \in \mathbb{N}}$ in such a way that the sequence $\left(\rho_{n}^{i}\left(\phi_{i}\right)\right)_{i \in \mathbb{N}}$ is summable in $H^{1, p}\left(\mathbb{R}^{N}\right)$, uniformly with respect to $n$, and that the sequence $\left(u_{n}-\sum_{i \in \mathbb{N}} \rho_{n}^{i}\left(\phi_{i}\right)\right)_{n \in \mathbb{N}}$ converges to zero in $L\left(p^{*}, q\right)$ for every index $q$ strictly greater than $p$.

Moreover we have that, for any pair of indexes $i$ and $j$, the two corresponding sequences of rescalings $\left(\rho_{n}^{i}\right)_{n \in \mathbb{N}}$ and $\left(\rho_{n}^{j}\right)_{n \in \mathbb{N}}$ are mutually diverging, that

$$
\sum_{i=1}^{+\infty}\left\|\phi_{i}\right\|_{1, p}^{p} \leq M
$$

where $M$ is the limit of $\left(\left\|u_{n}\right\|_{1, p}^{p}\right)_{n \in \mathbb{N}}$, and that the sequence $\left(u_{n}-\sum_{i \in \mathbb{N}} \rho_{n}^{i}\left(\phi_{i}\right)\right)_{n \in \mathbb{N}}$ converges to zero in $H^{1, p}\left(\mathbb{R}^{N}\right)$ if and only if (3) is an equality.

Remark. - The above result can be extended to cover the case $p=1$ provided the summability of the series is only asked in $L^{p^{*}}$ and (3) is replaced by the analogous estimate which only involves the $L^{p^{*}}$-norms. Of course the last part of the theorem becomes useless in such a case.

Remark. - Theorem 2 leads to a result like Lemma I.1 in [6], if one only looks to the approximation in the sense of the measures.

We need to introduce some more terminology to the aim of avoiding the necessity of using too many words for denoting the functions given by the above theorems. A weak limit point of a sequence like $\left(\rho_{n}\left(u_{n}\right)\right)_{n \in \mathbb{N}}$ in case (b) of Theorem 1 will be called a restored scale limit of the sequence $\left(u_{n}\right)_{n \in \mathbb{N}}$. Note that the restored scale limits of a sequence of functions are determined modulo a change of scale. In this sense, the functions $\phi_{i}$ introduced in Theorem 2 are (when they are not zero) exactly given by the nonzero restored scale limits of $\left(u_{n}\right)_{n \in \mathbb{N}}$, as follows from the fact that two sequences of rescalings corresponding to two different values of the index $i$ are mutually diverging. Let $U$ be a (bounded) subset of $H^{1, p}\left(\mathbb{R}^{N}\right)$. We shall say that $U$ has a bounded scale if, for every diverging sequence of rescalings $\left(\rho_{n}\right)_{n \in \mathbb{N}}$ and for every sequence $\left(u_{n}\right)_{n \in \mathbb{N}}$ of elements of $U$, the sequence of rescaled functions $\left(\rho_{n}\left(u_{n}\right)\right)_{n \in \mathbb{N}}$ weakly converges to zero in $L^{p^{*}}\left(\mathbb{R}^{N}\right)$. Note that a finite set obviously has a bounded scale and therefore every bounded subset of $H^{1, p}\left(\mathbb{R}^{N}\right)$ which is precompact with respect to 
the $L\left(p^{*}, q\right)$-norm, whatever $q$ is, has a bounded scale too. One can restate Theorem 1 in the following way.

THEOREM 3. - Let $p<N$ be given, let $U$ be a bounded subset of $H^{1, p}\left(\mathbb{R}^{N}\right)$ and let $q>p$. Then $U$ is precompact in $L\left(p^{*}, q\right)$ if and only if it has a bounded scale.

In all the above statements we could have considered the more general case in which the function $u$ has a gradient in a Lorentz Space $L\left(p_{1}, p_{2}\right)$ rather than in $L^{p}$. The proofs in the next section cover this more general case without any modification, as one sees from the remark after the proof of Theorem 1 which also shows how that result also holds in the optimal space $L\left(p_{1}^{*}, p_{2}\right)$ when $p_{2}=+\infty$. On the other hand, we can take in particular $q=p^{*}$ and we get in such a way the convergence and the precompactness in the $L^{p^{*}}$ space.

The statements are false in the optimal case, namely if one allows $q$ to be equal to $p$, as we shall see in the last section. For the case of general Lorentz spaces the analogous holds with the only exception of Marcinkiewicz spaces, as we have just pointed out.

\section{PROOF OF THE THEOREMS}

The proofs will be based on the following elementary estimate

$$
\left|u(\bar{x})-f_{B(\bar{x}, r)} u(x) d x\right| \leq c \int_{B(\bar{x}, r)} \frac{|\nabla u(x)|}{|x-\bar{x}|^{N-1}} d x
$$

where $f_{B(\bar{x}, r)} u(x) d x$ denotes the mean value of $u$ on the ball of center $\bar{x}$ and radius $r$ and $c$ is a positive constant. This can be easily proved by computing the variation of the mean values of $u$ on the various spheres. One can see from (4) that the function $\delta_{r} u$ which sends $\bar{x}$ in the first side of (4) is bounded by the convolution product of the function $|\nabla u|$ and of the function $|x|^{1-N}$ restricted to the ball centred at zero and of radius $r$. So the $L^{p}$-norm of $\delta_{r} u$ can be estimated by Young Formula with the product of the $L^{p}$-norm of $|\nabla u|$ and the $L^{1}$-norm of the other factor, which is given by a constant multiplied by $r$.

Proof of Theorem 1. - We firstly remark once more that we only have to care of the case $q=\infty$. In fact, by the Sobolev's Embedding in Lorentz Spaces, we know that the sequence $\left(u_{n}\right)_{n \in \mathbb{N}}$ is bounded in $L\left(p^{*}, p\right)\left(\mathbf{R}^{N}\right)$, 
so we see by interpolation, since $p<q$, that (a) holds provided $\left(u_{n}\right)_{n \in \mathbb{N}}$ converges to zero in the Marcinkiewicz space $L\left(p^{*}, \infty\right)$. So we have to show that, if (a) does not hold, and therefore the sequence does not converge to zero in $L\left(p^{*}, \infty\right)$, then (b) must be satisfied. We can pass to a subsequence in order to assume that the weak $L^{p^{*}}$ norm of all the functions $u_{n}$ is bounded from below by a positive constant $2 c$.

The lower bound in the Marcinkiewicz norm means that for every index $n$, we can find a nonnegligible measurable subset $Y_{n}$ of $\mathbb{R}^{N}$ such that

$$
\int_{Y_{n}}\left|u_{n}\right| \geq 2 c\left|Y_{n}\right|^{\frac{1}{p^{* /}}} .
$$

We can fix the modulus of $\rho_{n}$ in such a way that $\left|X_{n}\right|=\left|\left(\rho_{n}\right)^{-1}\left(Y_{n}\right)\right|=1$. From (5), taking into account that the rescalings $\rho_{n}$ preserve the weak $L^{p^{*}}$-norm, we have

$$
\int_{X_{n}}\left|\rho_{n}\left(u_{n}\right)\right| \geq 2 c .
$$

On the other hand, we have seen from (4) that the $L^{p}$-norms of the functions $\delta_{R}\left(\rho_{n}\left(u_{n}\right)\right)$ are bounded by the product of a constant times $R$, since the functions $\rho_{n}\left(u_{n}\right)$ are bounded in $H^{1, p}$. So we can fix $R$ conveniently small in order to have for every value of $n$

$$
\int_{X_{n}} \delta_{R}\left(\rho_{n}\left(u_{n}\right)\right) \leq c
$$

From (6) and (7), we see that we can find a point $x_{n}$ in $X_{n}$ such that $\left|\rho_{n}\left(u_{n}\right)\left(x_{n}\right)\right|-\delta_{R}\left(\rho_{n}\left(u_{n}\right)\right)\left(x_{n}\right) \geq c$, which trivially implies that

$$
\left|f_{B\left(x_{n}, R\right)} \rho_{n}\left(u_{n}\right)\right| \geq c .
$$

We finally fix the translation part of the rescalings $\rho_{n}$ in such a way to have $x_{n}=0$ for every $n$, then (8) shows that the sequence $\left(\rho_{n}\left(u_{n}\right)\right)_{n \in \mathbb{N}}$ has only nonzero weak limit points.

Remark. - Note that only an estimate on the weak $L^{p}$-norm of $\delta_{R}$ is necessary for (7). So the convergence of the sequence in the Marcinkiewicz space follows from a bound on the gradients with respect to the Marcinkiewicz norm. 
We do not need to give a proof of Theorem 3 which is a clear restatement of Theorem 1. In fact, if a set $U$ has a bounded scale, if $\left(u_{n}\right)_{n \in \mathbb{N}}$ is a sequence of elements of $U$ and if $\bar{u}$ is the weak limit of $\left(u_{n}\right)_{n \in \mathbb{N}}$, then the sequence $\left(u_{n}-\bar{u}\right)_{n \in \mathbb{N}}$ cannot satisfy condition (b) of Theorem 1, so (a) must hold and $\left(u_{n}\right)_{n \in \mathbb{N}}$ is therefore convergent.

Conversely, if we assume the statement of Theorem 3 and if we take the sequence $\left(u_{n}\right)_{n \in \mathbb{N}}$, we are in one of the following two situations. The sequence may have a nonzero weak limit point and then (b) holds, otherwise it weakly converges to zero and then if the convergence is strong (a) holds and if the convergence does not hold strongly then the set of the elements of the sequence cannot have a bounded scale and this means that (b) must be verified.

Analogously, we can very easily deduce Theorem 1 from Theorem 2, while, on the other hand, some work is needed for the proof of Theorem 2. The argument is a more complicated variant of the proof of the main result in [11], which can be read as an example of a simple case where the estimates developed below are not necessary.

Proof of Theorem 2. - Assume that $\left(u_{n}\right)_{n \in \mathbb{N}}$ does not converge to zero in $L\left(p^{*}, q\right)$ for every $q$ greater than $p$, if not we take $\phi_{i}=0$ for every $i$ and we have nothing to prove. Then we know by Theorem 1 that we can select a subsequence (which we shall still denote by $\left(u_{n}\right)_{n \in \mathbb{N}}$ ) and a sequence of rescalings $\left(\rho_{n}\right)_{n \in \mathbb{N}}$ such that the sequence of rescaled functions $\left(\rho_{n}\left(u_{n}\right)\right)_{n \in \mathbb{N}}$ weakly converges to a nonzero limit and we denote such a limit by $\phi_{1}$. We shall choose $\phi_{1}$ to have a $H^{1, p}$-norm greater or equal to one half of the supremum of the norms of the restored scale limits of the sequence $\left(u_{n}\right)_{n \in \mathbb{N}}$. Of course we have

$$
\left\|\phi_{1}\right\|_{1, p}^{p} \leq M
$$

Then we take as $\rho_{n}^{1}$ the inverse of $\rho_{n}$ for large values of $n$ and we set $u_{n}^{1}=u_{n}-\rho_{n}^{1}\left(\phi_{1}\right)$. Then we reapply the same procedure to the new sequence $\left(u_{n}^{1}\right)_{n \in \mathbb{N}}$ and we can find a new subsequence, which we shall again denote by the same symbol as before, and a new sequence of rescalings $\left(\rho_{n}\right)_{n \in \mathbb{N}}$ such that $\left(\rho_{n}\left(u_{n}^{1}\right)\right)_{n \in \mathbb{N}}$ has a weak limit $\phi_{2}$, with a $H^{1, p}$-norm greater or equal to one half of the supremum of the norms of the restored scale limits of the sequence $\left(u_{n}^{1}\right)_{n \in \mathbb{N}}$. We shall denote by $\rho_{n}^{2}$ the inverse of this new $\rho_{n}$, for $n$ large. One sees trivially that the two sequences of rescalings $\left(\rho_{n}^{1}\right)_{n \in \mathbb{N}}$ and $\left(\rho_{n}^{2}\right)_{n \in \mathbb{N}}$ must be mutually diverging (unless $\phi_{2}=0$, in which case we can define the rescalings $\rho_{n}$ arbitrarily). 
When we replace $\left(u_{n}^{1}\right)_{n \in \mathbb{N}}$ by its subsequence we keep the first term $u_{1}^{1}$. We have the analogous of (9)

$$
\left\|\phi_{1}\right\|_{1, p}^{p}+\left\|\phi_{2}\right\|_{1, p}^{p} \leq M
$$

To the aim of proving the above estimate we first observe that, by the mutual divergence of $\left(\rho_{n}^{1}\right)_{n \in \mathbb{N}}$ and $\left(\rho_{n}^{2}\right)_{n \in \mathbb{N}}$, in correspondence of any given constant $a>1$, we have for $n$ large

$$
\begin{aligned}
a^{-1}\left\|\rho_{n}^{1}\left(\phi_{1}\right)+\rho_{n}^{2}\left(\phi_{2}\right)\right\|_{1, p}^{p} & \leq\left\|\phi_{1}\right\|_{1, p}^{p}+\left\|\phi_{2}\right\|_{1, p}^{p} \\
& \leq a\left\|\rho_{n}^{1}\left(\phi_{1}\right)+\rho_{n}^{2}\left(\phi_{2}\right)\right\|_{1, p}^{p}
\end{aligned}
$$

We shall fix the values of $\rho_{n}^{2}$ for small values of $n$ in such a way to have (11) satisfied by every $n$ in correspondence of $a=2$. Then, for $i=1,2$, we fix an element $\psi_{i}$ in the dual space $H^{-1, p^{\prime}}$ such that $\left\|\psi_{i}\right\|_{-1, p^{\prime}}=\left\|\phi_{i}\right\|_{1, p}^{p-1}$ and $\int \phi_{i} \psi_{i}=\left\|\phi_{i}\right\|_{1, p}^{p}\left(\psi_{i}=\operatorname{div}\left(\left|\nabla \phi_{i}\right|^{p-2} \nabla \phi_{i}\right)\right)$. In the same way as before, we have for $n$ large

$$
\begin{aligned}
a^{-1}\left\|\left(\rho_{n}^{1}\right)^{\prime}\left(\psi_{1}\right)+\left(\rho_{n}^{2}\right)^{\prime}\left(\psi_{2}\right)\right\|_{-1, p^{\prime}}^{p^{\prime}} & \leq\left\|\psi_{1}\right\|_{-1, p^{\prime}}^{p^{\prime}}+\left\|\psi_{2}\right\|_{-1, p^{\prime}}^{p^{\prime}} \\
& \leq a\left\|\left(\rho_{n}^{1}\right)^{\prime}\left(\psi_{1}\right)+\left(\rho_{n}^{2}\right)^{\prime}\left(\psi_{2}\right)\right\|_{-1, p^{\prime}}^{p^{\prime}}
\end{aligned}
$$

By combining the above estimates and by using (2), we find

$$
\begin{aligned}
\left\|\phi_{1}\right\|_{1, p}^{p}+\left\|\phi_{2}\right\|_{1, p}^{p} & =\int \phi_{1} \psi_{1}+\int \phi_{2} \psi_{2} \\
& =\lim _{n}\left(\int\left(\rho_{n}^{1}\right)^{-1}\left(u_{n}\right) \psi_{1}+\int\left(\rho_{n}^{2}\right)^{-1}\left(u_{n}\right) \psi_{2}\right) \\
& =\lim _{n} \int u_{n}\left(\left(\rho_{n}^{1}\right)^{\prime}\left(\psi_{1}\right)+\left(\rho_{n}^{2}\right)^{\prime}\left(\psi_{2}\right)\right) \\
& \leq M^{\frac{1}{p}}\left(\left\|\left(\rho_{n}^{1}\right)^{\prime}\left(\psi_{1}\right)+\left(\rho_{n}^{2}\right)^{\prime}\left(\psi_{2}\right)\right\|_{-1, p^{\prime}}^{p^{\prime}}\right)^{\frac{1}{p^{\prime}}} \\
& \leq M^{\frac{1}{p}} a^{\frac{1}{p^{\prime}}}\left(\left\|\phi_{1}\right\|_{1, p}^{p}+\left\|\phi_{2}\right\|_{1, p}^{p}\right)^{\frac{1}{p^{\prime}}}
\end{aligned}
$$


which gives

$$
\left\|\phi_{1}\right\|_{1, p}^{p}+\left\|\phi_{2}\right\|_{1, p}^{p} \leq a^{p-1} M
$$

and therefore, by the arbitrariness of $a$, we obtain (10). We must now iterate this process. We set $u_{n}^{2}=u_{n}^{1}-\rho_{n}^{2}\left(\phi_{2}\right)$ and we take as $\phi_{3}$ a restored scale limit of a suitable subsequence of $\left(u_{n}^{2}\right)_{n \in N}$, which keeps the first two terms of the previous one, whose norm is greater or equal than one half of the supremum of all the norms of the restored scale limits of $\left(u_{n}^{2}\right)_{n \in \mathrm{N}}$. In the same way we define the following limits $\phi_{i}$ and the following sequences $\left(u_{n}^{i}\right)_{n \in \mathrm{N}}$. We can generalize, by repeating the same proofs, the estimates (11) and (12) in such a way that, for every finite subset $J$ of $\mathbb{N}$, in correspondence of any given constant $a>1$, we have for $n$ sufficently large

$$
\begin{gathered}
a^{-1}\left\|\sum_{i \in J} \rho_{n}^{i}\left(\phi_{i}\right)\right\|_{1, p}^{p} \leq \sum_{i \in J}\left\|\phi_{i}\right\|_{1, p}^{p} \leq a\left\|\sum_{i \in J} \rho_{n}^{i}\left(\phi_{i}\right)\right\|_{1, p}^{p} \\
a^{-1}\left\|\sum_{i \in J}\left(\rho_{n}^{i}\right)^{\prime}\left(\psi_{i}\right)\right\|_{-1, p^{\prime}}^{p^{\prime}} \leq \sum_{i \in J}\left\|\psi_{i}\right\|_{-1, p^{\prime}}^{p^{\prime}} \leq a\left\|\sum_{i \in J}\left(\rho_{n}^{i}\right)^{\prime}\left(\psi_{i}\right)\right\|_{-1, p^{\prime}}^{p^{\prime}}
\end{gathered}
$$

As we have said before, we can assume that the last two estimates hold for every $n$ for $a=2$. We finally substitute the sequence $\left(u_{n}\right)_{n \in \mathrm{N}}$ with a diagonal selection of successive subsequences which we have extracted for every value of $i$. The fact that we have always kept the first terms of a sequence when we have sobstituted it with a subsequence makes the terms of the diagonal selection belong to all the successive subsequences corresponding to the values of the index $i$. Again we have as before, for any finite subset $J$ of $\mathbb{N}$,

$$
\begin{aligned}
\sum_{i \in J}\left\|\phi_{i}\right\|_{1, p}^{p} & =\lim _{n} \int u_{n} \sum_{i \in J}\left(\rho_{n}^{i}\right)^{\prime}\left(\psi_{i}\right) \\
& \leq M^{\frac{1}{p}}\left(\left\|\sum_{i \in J}\left(\rho_{n}^{i}\right)^{\prime}\left(\psi_{i}\right)\right\|_{-1, p^{\prime}}^{p^{\prime}}\right)^{\frac{1}{p^{\prime}}} \\
& \leq M^{\frac{1}{p}} a^{\frac{1}{p^{\prime}}}\left(\sum_{i \in J}\left\|\phi_{i}\right\|_{1, p}^{p}\right)^{\frac{1}{p^{\prime}}}
\end{aligned}
$$

and, consequentely, by the arbitrariness of $a$

$$
\sum_{i \in J}\left\|\phi_{i}\right\|_{1, p}^{p} \leq M
$$


which gives (3). The uniform summability of $\left(\rho_{n}^{i}\right)_{i \in \mathbb{N}}$ is then an obvious consequence of (13) and (3). In order to prove the convergence to zero of $\left(u_{n}-\sum_{i \in \mathbb{N}} \rho_{n}^{i}\left(\phi_{i}\right)\right)_{n \in \mathbb{N}}$ in $L\left(p^{*}, q\right)$ for $q$ strictly greater than $p$, we only have to show that the sequence has no nonzero restored scale limit and then apply Theorem 1 . To this aim, let $\phi$ denote a restored scale limit of $\left(u_{n}-\sum_{i \in \mathbb{N}} \rho_{n}^{i}\left(\phi_{i}\right)\right)_{n \in \mathbb{N}}$, fix an integer $h$ and, for every $n$, split $u_{n}-\sum_{i \in \mathbb{N}} \rho_{n}^{i}\left(\phi_{i}\right)$ in the sum of $u_{n}^{h}$ and $\sum_{i=h+1}^{+\infty} \rho_{n}^{i}\left(\phi_{i}\right)$. Let $\phi^{\prime}$ be the weak limit of a suitable subsequence of $\left(u_{n}^{h}\right)_{n \in \mathbb{N}}$, suitably rescaled term by term, and $\phi^{\prime \prime}$ the weak limit of the corresponding rescaled subsequence of $\left(\sum_{i=h+1}^{+\infty} \rho_{n}^{i}\left(\phi_{i}\right)\right)_{n \in \mathbb{N}}$, chosen in such a way to have $\phi=\phi^{\prime}+\phi^{\prime \prime}$. By construction we have $\left\|\phi^{\prime}\right\|_{1, p} \leq 2\left\|\phi_{h+1}\right\|_{1, p}$ and by the weak semicontinuity of the norm and by (13) we have $\left\|\phi^{\prime \prime}\right\|_{1, p} \leq\left(\sum_{i=h+1}^{+\infty}\left\|\phi_{i}\right\|_{1, p}^{p}\right)^{\frac{1}{p}}$.

By (3) we see that the right sides of the two last inequalities both converge to zero as $h$ tends to infinity. This implies, by the arbitrariness of $h$, that we must have $\phi=0$ and then the desired convergence of $\left(u_{n}-\sum_{i \in \mathbb{N}} \rho_{n}^{i}\left(\phi_{i}\right)\right)_{n \in \mathbb{N}}$. For what concernes the $H^{1, p}\left(\mathbb{R}^{N}\right)$-convergence we observe that by (13) and (14) we obtain

$$
\begin{gathered}
\lim _{n}\left\|\sum_{i=1}^{+\infty} \rho_{n}^{i}\left(\phi_{i}\right)\right\|_{1, p}^{p}=\sum_{i=1}^{+\infty}\left\|\phi_{i}\right\|_{1, p}^{p} \\
\lim _{n}\left\|\sum_{i=1}^{+\infty}\left(\rho_{n}^{i}\right)^{\prime}\left(\psi_{i}\right)\right\|_{-1, p^{\prime}}^{p^{\prime}}=\sum_{i=1}^{+\infty}\left\|\psi_{i}\right\|_{-1, p^{\prime}}^{p^{\prime}}=\sum_{i=1}^{+\infty}\left\|\phi_{i}\right\|_{1, p}^{p} .
\end{gathered}
$$

So, when the $H^{1, p}\left(\mathbb{R}^{N}\right)$-convergence holds, we see from (17) that we must have the equality in (3), while, if such an equality holds, for every $n$ we have two vectors in $H^{1, p}\left(\mathbb{R}^{N}\right)$, namely $u_{n}$ and $\sum_{i \in \mathbb{N}} \rho_{n}^{i}\left(\phi_{i}\right)$, which tend both to have a norm equal to $M^{\frac{1}{p}}$ and a linear form, induced by the dual vector $\sum_{i \in \mathbb{N}}\left(\rho_{n}^{i}\right)^{\prime}\left(\phi_{i}\right)$, whose norm tends to $M^{\frac{1}{p^{p}}}$. Moreover the linear form computed in $\sum_{i \in \mathbb{N}} \rho_{n}^{i}\left(\phi_{i}\right)$ tends to $M$, as one can easily check by using the 
uniform convergence of the two sums and the mutual divergence of two different sequences of rescalings, and computed in $u_{n}$ tends to $M$ again as follows from (15).

By the uniform convexity of the $L^{p}$ norms this fact implies that the difference $\left(u_{n}-\sum_{i \in \mathbb{N}} \rho_{n}^{i}\left(\phi_{i}\right)\right)_{n \in \mathbb{N}}$ converges to zero in $H^{1, p}\left(\mathbb{R}^{N}\right)$.

\section{FURTHER REMARKS AND COUNTEREXAMPLES}

Some particular cases in which the statements of the above theorems become simpler will be considered in the beginning of this section. One of this will be the locally compact case in which we know that the sequence $\left(u_{n}\right)_{n \in \mathbb{N}}$ is also bounded in the $L^{s}$-norm, when $s$ is an integer greater than $p^{*}$. Then we can easily check that, if $i$ is an index such that $\left(\rho_{n}^{i}\right)_{n \in \mathbb{N}}$ is diverging by concentration and if $\phi_{i}$ is different from zero, then $\left(\rho_{n}^{i}\left(\phi_{i}\right)\right)_{n \in \mathbb{N}}$ blows up in the $L^{s}$-norm, even modulo bounded perturbations in the $L^{p^{*}}$ norm, since a big part of the norm of $\rho_{n}^{i}\left(\phi_{i}\right)$ is taken by integrating on a set whose measure tends to zero with respect to $n$. Since the distance between $u_{n}$ and $\rho_{n}^{i}\left(\phi_{i}\right)$ is clearly bounded in $L^{p^{*}}$, this fact leads to the blow up of $\left(u_{n}\right)_{n \in \mathbb{N}}$ in $L^{s}$, an obvious contradiction. So we see that in Theorem 2 we only have to consider sequences of rescalings which are diverging only by translation or by vanishing. Conversely, the sequence $\left(\rho_{n}\right)_{n \in \mathbb{N}}$ considered in Theorem 1 can only diverge by translation or by concentration and the boundedness of the scale of the sets which are bounded in $L^{s}$, besides being bounded in $H^{1, p}$, only needs to be checked with sequences of rescalings diverging by translation or by concentration.

Let us consider now the behaviour of $\left(u_{n}\right)_{n \in \mathbb{N}}$ in $L^{r}$, when $r$ is any number strictly contained between $p^{*}$ and $s$. We claim that the approximation shown in Theorem 2 also holds in $L^{r}$. In fact we just have to know that the sequence $\left(\sum_{i \in \mathbb{N}} \rho_{n}^{i}\left(\phi_{i}\right)\right)_{n \in \mathbb{N}}$ can be taken bounded in $L^{s}$ and to apply a simple interpolation argument. The above mentioned boundedness follows by similar arguments to those in the proof of Theorem 2. In fact, we can chose the rescalings in such a way to have the analogous of (16), namely

$$
\left\|\sum_{i \in J} \rho_{n}^{i}\left(\phi_{i}\right)\right\|_{s}^{s} \leq 2 \sum_{i \in J}\left\|\rho_{n}^{i}\left(\phi_{i}\right)\right\|_{s}^{s} \leq 2^{s} M_{s}
$$

where $M_{s}^{\frac{1}{s}}$ is the limit of $L^{s}$-norm of $u_{n}$, by the same arguments used for that proof, with obvious differences.

Vol. $12, n^{\circ} 3-1995$. 
(One just has to take as $\psi_{i}$ the appropriate dual function of $\phi_{i}$ with respect to the $L^{s}$ structure, namely $\psi_{i}=\left|\phi_{i}\right|^{s-2} \phi_{i}$, and to prove, for any subset $J$ of $\mathbb{N}$ and for any integer $n$, the analogous of (13)-(14) (for $a=2$ ) as the first inequality in (19) and finally of (15), namely

$$
\begin{aligned}
\sum_{i \in J}\left\|\rho_{n}^{i}\left(\phi_{i}\right)\right\|_{s}^{s} & \leq \lim _{n} \int u_{n} \sum_{i \in J}\left(\rho_{n}^{i}\right)^{\prime}\left(\psi_{i}\right) \\
& \leq M_{s}^{\frac{1}{s}}\left\|\sum_{i \in J}\left(\rho_{n}^{i}\right)^{\prime}\left(\psi_{i}\right)\right\|_{s^{\prime}} \\
& \leq M_{s}^{\frac{1}{s}}\left(2 \sum_{i \in J}\left\|\rho_{n}^{i}\left(\phi_{i}\right)\right\|_{s}^{s}\right)^{\frac{1}{s^{\prime}}}
\end{aligned}
$$

which gives the last inequality in (19).)

Note that the analogous of (3) or of (16) for the $L^{s}$-norms cannot hold, of course, for the functions $\phi_{i}$ nonrescaled since those functions are only determined modulo a rescaling which does not leave invariant the $L^{s}$-norm. Finally, from the uniform summability of the series $\sum_{i} \rho_{n}^{i}\left(\phi_{i}\right)$ in $L^{p^{*}}$ and the uniform bound on all the partial sums given in (19), one can deduce by interpolation the uniform summability also in $L^{r}$. If for some index $i$ the sequence of rescalings $\left(\rho_{n}^{i}\right)_{n \in \mathbb{N}}$ is diverging by vanishing, then the term $\left(\rho_{n}^{i}\left(\phi_{i}\right)\right)_{n \in \mathbb{N}}$ is infinitesimal in $L^{r}$ and can therefore be erased from the approximating series $\sum_{i} \rho_{n}^{i}\left(\phi_{i}\right)$. By its uniform summability we can also simultaneously erase all such terms and therefore we can assume, as far as only the $L^{r}$ approximation is involved, that the sequences $\left(\rho_{n}^{i}\right)_{n \in \mathbb{N}}$ can only diverge by translation.

The converse properties are of course true if one considers the nonvanishing case given by a bound in $L^{s}$ with $s<p^{*}$. In such a case the sequence $\left(\rho_{n}^{i}\right)_{n \in \mathrm{N}}$ cannot diverge by vanishing and the indexes for which they are diverging by concentration can be forgotten if one wants to approximate $\left(u_{n}\right)_{n \in \mathbb{N}}$ only in the $L^{r}$-norm, for some $s<r<p^{*}$.

The divergence by translation can finally also be excluded in some particular cases as when one looks to suitably symmetric functions or when the support of the functions $u_{n}$ is contained in a set of finite measure, which also is an obvious nonvanishing case. When the three forms of diverging sequences of rescalings are simultaneously avoided one must have that at 
most one of the functions $\phi_{i}$ can be different from zero, indeed, if $i$ and $j$ are two indexes such that $\phi_{i}$ and $\phi_{j}$ are both different from zero then at least one of two corresponding sequences of rescaling must be diverging, since the two sequences are mutually diverging. Moreover we can take all the rescalings $\rho_{n}^{i}$ equal to the identity and so the sequence $\left(u_{n}\right)_{n \in \mathbb{N}}$ strongly converges to $\phi_{i}$.

This situation happens, for instance, as we have already said, when we consider a set of radially symmetric functions or a set of functions with a support contained in a given set of finite measure and we look for a limit in $L^{r}$ of a sequence $\left(u_{n}\right)_{n \in \mathrm{N}}$ which is bounded in the Sobolev Space $H^{1, p}$ and in $L^{s}$ with $r$ included in the open interval of extrema $p^{*}$ and $q$. The last bound is of course implicit in the case of a domain of finite measure when $r<p^{*}$, the situation of Rellich Theorem.

Let us come now to examinate the case of the optimal embedding corresponding to $q=p$. We shall indicate a simple example which shows that the three theorems proved in this section do not hold in such a case. In fact we shall define a bounded sequence of $H^{1, p}\left(\mathbb{R}^{N}\right)$ which has no nonzero restored scale limits and which does not converge to zero in $L\left(p^{*}, p\right)$. To this aim, fix a positive function $\phi$ in $H^{1, p}\left(\mathbb{R}^{N}\right)$ and take, in order to simplify the argument, the support of $\phi$ contained in the annulus centred in zero and with radii equal to 1 and 2 . For every integer number $n$, we choose as $u_{n}$ the following function: for $i=1,2, \ldots, n$ we take the function $v_{i}$ given by the rescaling of $\phi$ which keeps invariant the $H^{1, p}$-norm with center zero and modulus $2^{i}$, then we define as $u_{n}$ the sum of the $n$ functions $v_{i}$ normalized by the product with the constant $n^{-\frac{1}{p}}$. Since the functions $v_{i}$ have disjoint supports, we easily see that for every $n$ we have $\left\|u_{n}\right\|_{1, p}^{p}=\|\phi\|_{1, p}^{p}$ and $\left\|u_{n}\right\|_{p^{*}}^{p^{*}}=n^{1-\frac{p^{*}}{p}}\|\phi\|_{p^{*}}^{p^{*}}$. So the sequence $\left(u_{n}\right)_{n \in \mathrm{N}}$ converges to zero in $L^{p^{*}}$ and therefore it cannot have nonzero restored scale limits. It must clearly converge to zero also in $L\left(p^{*}, q\right)$ for every $q>p$, as follows by interpolation since the sequence is bounded in $L\left(p^{*}, p\right)$. We claim that $\left(u_{n}\right)_{n \in \mathbb{N}}$ does not converge to zero in $L\left(p^{*}, p\right)$ and therefore Theorem 2 does not hold with $q=p$. In fact, if this were the case, then $u_{n}^{p}$ would converge to zero in $L\left(\frac{p^{*}}{p}, 1\right)=L\left(\frac{N}{N-p}, 1\right)$. This conclusion does not hold because, if we take the scalar product of $u_{n}^{p}$ with the function $\frac{1}{|x|^{p}}$, which is in $L\left(\frac{N}{p}, \infty\right)=L\left(\left(\frac{p^{*}}{p}\right)^{\prime}, \infty\right)$, we have 


$$
\begin{aligned}
\int_{\mathbb{R}^{N}} u_{n}^{p} \frac{1}{|x|^{p}} & =n^{-\frac{1}{p} p} \int_{\mathbb{R}^{N}}\left(\sum_{i=1}^{n} v_{i}^{p} \frac{1}{|x|^{p}}\right) \\
& \left.=n^{-1} \sum_{i=1}^{n} \int_{\mathbb{R}^{N}}\left(2^{i \frac{N-p}{p}} \phi\left(2^{i} x\right)\right)^{p} \frac{1}{|x|^{p}}\right) d x \\
& \left.=n^{-1} \sum_{i=1}^{n} 2^{i(N-p)} \int_{\mathbb{R}^{N}}\left(\phi\left(2^{i} x\right)\right)^{p} \frac{2^{i p}}{\left|2^{i} x\right|^{p}}\right) 2^{-N i} d\left(2^{i} x\right) \\
& =\int_{\mathbb{R}^{N}} \phi(x) \frac{1}{|x|^{p}} d x .
\end{aligned}
$$

One can see in this example that the functions $u_{n}$ tend to "distribute $\phi$ on a wide range of scales" by breaking it in $n$ different parts and by rescaling each one of them in a different way. This is exactly what cannot happen in the nonoptimal cases in which a sequence of functions bounded in the Sobolev Space which does not converge to zero has to have some part "concentrated on some precise scale". Such a scale can change for different values of the index but it can always be localized and "normalized" by a suitable rescaling.

This example can be extended to cover the case of a sequence of functions with the gradients bounded in a Lorentz Space $L\left(p_{1}, p_{2}\right)$, when $p_{2} \neq 0$. In such a case one can repeate the same construction as above, by only paing attention to fix a suitably consistent difference of scale between two different functions $v_{i}$, rather than to consider two consecutive ones of those functions only rescaled by a factor two. Then one can check, by looking directly to the definition of Lorentz norm, that the normalization factor of $u_{n}$ is $n^{-\frac{1}{p_{2}}}$, then the sequence turns out to be infinitesimal in $L\left(p_{1}^{*}, q\right)$ for $q>p_{2}$ and not when $q=p_{2}$.

We point out that this kind of example only depends on a simple property of the optimal embedding. In fact, we have shown that, when $p_{2} \neq 0$, a sequence can be found which is bounded in $H^{1,\left(p_{1}, p_{2}\right)}\left(\mathbb{R}^{N}\right)$, converges to zero in $L\left(p_{1}^{*}, \infty\right)$ and does not converge to zero in $L\left(p_{1}^{*}, p_{2}\right)$. The invariance of the norm of $L\left(p_{1}^{*}, \infty\right)$ with respect to the rescalings implies that all the restored scale limits of the sequence must be zero. Since the sequence is not infinitesimal in $L\left(p_{1}^{*}, p_{2}\right)$, Theorem 2 cannot be extended to cover the case of the optimal secundary index.

To the aim of checking if we have in some concrete variational problems, in which the $L\left(p^{*}, p\right)$-norm is involved, a minimizing sequence which behaves as the one in the above example, let us consider the well known problem

$$
-\Delta u=u^{\alpha}
$$


We look for (positive) solutions to (P) in $H_{0}^{1}\left(\mathbb{R}^{N}\right)$. The well known Pohozaev Identity $[10]$ shows that regular solutions to $(\mathrm{P})$ can only appear for $\alpha=\frac{N+2}{N-2}$ and, in such a case, one looks for a ground state solution by minimizing $\|u\|_{1,2}^{2}$ on the set of the functions $u$ such that $\|u\|_{2^{*}}^{2^{*}} \geq 1$. One can easily show that such a minimum is achieved by using a result like Theorem 2 and taking advantage of the fact that the constraint which determines the set where the $H^{1}$-norm has to be minimized is continuous in $L^{2^{*}}$. (The proof in [7] that dichotomy cannot occur is enough to show that only one of the functions $\phi_{i}$ given by Theorem 2 in correspondence of a minimizing sequence of the constrained variational problem is different from zero.)

The analogous of this problem in which one has to consider a constraint which only depends on the $L\left(2^{*}, 2\right)$-norm is the linear eigenvalue problem

$$
-\Delta u=\lambda \frac{u}{|x|^{\alpha}} \text {. }
$$

Note that in [7] this problem is presented as a case in which the concentration-compactness inequality does not hold. (See [3] and [2] for the study of problems which can be regarded as the natural extension of (P) to cases which involve the embedding of $H_{0}^{1}$ in stronger Lorentz Spaces than $L^{2^{*}}$.) We shall now show that (LP) has no positive solutions in $H_{0}^{1}(\Omega)$ if $\Omega=\mathbb{R}^{N}$ or if $\Omega$ is an angular domain with vertex in zero.

The proof can be achieved in three steps. Firstly we observe that a Pohozaev type identity (see [2]) shows that we must have $\alpha=2$. Then we claim that if we have a pair $(\bar{\lambda}, \bar{u})$ which solves (LP), then $\bar{\lambda}$ must be equal to the "first eigenvalue" $\lambda_{1}$ given by the infimum of the value of $\|u\|_{1,2}^{2}$ taken on the set of the functions $u$ such that $\int \frac{u^{2}}{|x|^{2}} \geq 1$. (Note that $\lambda_{1}$ has not to be an eigenvalue of (LP) but only a point of the spectrum since the operator which sends $u$ in $\frac{u}{|x|^{2}}$ is not compact from $H^{1}$ in $\left.H^{-1}\right)$. This fact is intuitively obvious, nevertheless we prefer to prove it rigorously. If, by contradiction, we assume $\bar{\lambda}>\lambda_{1}$, we can certainly find a function $\bar{v}$ such that

$$
\int \frac{\bar{v}^{2}}{|x|^{2}} \geq 1
$$

and

$$
\|\bar{v}\|_{1,2}^{2}<\bar{\lambda}
$$


By multipling $\bar{v}$ by a coefficient slightly greater than one, we can always assume that the inequality (20) is strict. So we can choose a function $\nu$, bounded and with a compact support, obtained by truncation from the function $\frac{1}{|x|^{2}}$, which preserves (20), namely such that

$$
\int \nu \bar{v}^{2} \geq 1
$$

Then we consider the first eigenvalue $\lambda_{1}^{0}$ (which exists since the operator which sends $u$ in $\nu u$ is compact from $H^{1}$ in $H^{-1}$, see, for instance, [8]) of the linear eigenvalue problem

$$
\left(\mathrm{LP}_{0}\right)-\Delta u=\lambda \nu u .
$$

By a test with $\bar{v}$ and by using the variational characterization of the eigenvalues, we deduce from (21) and (22) that $\lambda_{1}^{0}<\bar{\lambda}$.

Let $\phi$ be an eigenvector of $\left(\mathrm{LP}_{0}\right)$ corresponding to $\lambda_{1}^{0}$. By the strong maximum principle we see that $\bar{u}$ and $\phi$ are strictly positive in every point of $\Omega$. By using the fact that $\bar{u}$ and $\phi$ are eigenvectors of (LP) and (LP $P_{0}$, we have

$$
\int(-\Delta \phi) \bar{u}=\int \lambda_{1}^{0} \nu \phi \bar{u}<\int \bar{\lambda} \frac{\bar{u}}{|x|^{2}} \phi=\int(-\Delta \bar{u}) \phi,
$$

an obvious contradiction. So we must have $\bar{\lambda}=\lambda_{1}$ and $\lambda_{1}$ must therefore be an eigenvalue of (LP). By a well known variational argument (see [8]) we know that its multiplicity must be equal to one while, on the other hand, since (LP) is invariant with respect to the rescalings, we see that if we rescale an eigenfunction we obtain another eigenfunction. The combination of the two facts shows that the eigenfunctions corresponding to $\lambda_{1}$ must be "selfrescaled" and therefore they can only be weakly summable. The computations for the radial case show that this is actually the case when $\Omega=\mathbb{R}^{N}$; in fact one finds that the eigenfunctions with a gradient weakly in $L^{2}$ must be equal to a constant multiplied by the selfrescaled function $|x|^{\frac{2-N}{2}}$ which only belongs to the weak $H^{1}$-space. This analysis shows that the minimization process tends to distribute the function equally on all the possible scales, as happens in the counterexample in this section.

\section{REFERENCES}

[1] A. Alvino, P. L. Lions and G. Trombetri, On optimization problems with prescribed rearrangements, Nonlinear Analysis, T.M.A., Vol. 13, 1989, pP. 185-220. 
[2] D. Fortunato, E. Jannelli and S. Solimini, in preparation.

[3] V. Glaser, A. Martin, H. Grosse and W. Thirring, A family of optimal conditions for the absence of bound states in a potential, in "Studies in Mathematical Physics", E. H. Lieb, B. Simon and A. S. Wightman eds., Princeton University Press, 1976, pp. 169-194.

[4] P. L. Lions, The Concentration-Compactness Principle in the Calculus of Variations. The locally compact case-Part I, Ann. Inst. H. Poincare, Vol. 1, 1984, pp. 109-145.

[5] P. L. Lions, The Concentration-Compactness Principle in the Calculus of Variations. The locally compact case-Part II, Ann. Inst. H. Poincare, Vol. 1, 1984, pp. 223-283.

[6] P. L. Lions, The Concentration-Compactness Principle in the Calculus of Variations. The limit case-Part I, Rev. Mat. Iberoamericana, Vol. 1, No. 1, 1985, pp. 145-201.

[7] P. L. Lions, The Concentration-Compactness Principle in the Calculus of Variations. The limit case-Part II, Rev. Mat. Iberoamericana, Vol. 1, No. 2, 1985, pp. 45-121.

[8] A. Manes and A. M. Micheletti, Un' estensione della teoria variazionale classica degli autovalori per operatori ellittici del secondo ordine, Boll. UMI, 1973, pp. 285-301.

[9] R. O'NeIL, Convolution operators and $L(p, q)$ spaces, Duke Math. J., Vol. 30, 1963, pp. 129-142.

[10] S. Pohozaev, Eigenfunction of the equation $\Delta u+\lambda f(u)=0$, Soviet Math. Doklady, Vol. 6, 1965, pp. 1408-1411.

[11] M. STRUWE, A global compactness result for elliptic boundary value problems involving limiting nonlinearities, Math. Z., Vol. 187, 1984, pp. 511-517. 